\title{
Osborn Equation and Irrelevant Operators
}

\author{
Adam Schwimmer ${ }^{a}$ and Stefan Theisen ${ }^{b}$ \\ ${ }^{a}$ Weizmann Institute of Science, Rehovot 76100, Israel \\ ${ }^{b}$ Max-Planck-Insitut für Gravitationsphysik, Albert-Einstein-Institut, 14476 Golm, Germany
}

\begin{abstract}
The structure of the Osborn ("Local Renormalization Group") Equation in the presence of integer dimensional irrelevant operators is studied. We argue that the consistency of the anomalous part of the generating functional requires a beta-function for the metric. The modified form of the Weyl anomalies is calculated.
\end{abstract}




\section{Introduction}

Integer dimensional primary operators in Conformal Field Theories (CFT) give rise to conformal anomalies [1]. While at separate space-time points the correlators of these operators are well defined, at coincident points the singularity needs a regulator. As a consequence there is an irremovable violation of the conformal Ward identities. The singularities involved are typically logarithmic, leading to the so-called "type B" conformal anomalies [2]. In particular situations involving identities valid in special space-time dimensions, the logarithms are converted into integer power singularities without a scale ("type A" conformal anomalies) [3].

Higher order correlators of integer dimensional operators produce increasing powers of logarithms. The recursive nature of the correlators related to their analytic structure in momentum space reduce the higher powers of logarithms to the independent, universal information contained in the single logarithm term in each correlator.

This structure is summarized in the Osborn Equation. While the equation can be used to describe the perturbative renormalization group flow in a CFT perturbed by relevant operators, in the present paper we will use it strictly in the CFT: the space-time dependent couplings will be considered as sources of the primary operators. The coefficients of the Taylor expansion of the partition function around zero sources are the CFT correlators.

We give now a brief description of the Osborn Equation [4]. ${ }^{1}$ Consider in a CFT relevant and marginal primaries $\mathcal{O}_{i}$ with integral dimensions $\Delta_{i}$. The operators $\mathcal{O}_{i}$ are coupled to space-time dependent sources $J_{i}(x)$. In the list of primary operators we include the energymomentum tensor $T_{\mu \nu}(x)$ coupled to the background metric $g_{\mu \nu}(x)$. All other operators which we will consider in this work are scalars. The connected generating functional $W$ depends on $J_{i}(x)$ and $g_{\mu \nu}(x)$. The local Weyl variation operator acts on $W$ as

$$
\delta_{\sigma} W=\int d^{d} x\left\{2 \sigma(x) g_{\mu \nu}(x) \frac{\delta}{\delta g_{\mu \nu}(x)}+\sum_{i} \beta_{i}\left(\left\{J_{j}\right\}, g_{\mu \nu} ; \sigma\right) \frac{\delta}{\delta J_{i}(x)}\right\} W
$$

where the first term represents the canonical Weyl transformation of the metric and the second term involves the local "beta-functions" of the sources (couplings) linear in the infinitesimal Weyl parameter $\sigma(x)$ and its derivatives.

We remark that the non-classical part of the beta-function, i.e. the part which contains information about logarithms in correlation functions, arises from the action of the Weyl transformation on the anomalous part of the generating functional. Equivalently, the correlation functions satisfy the $S O(d, 2)$ (the conformal group in $d$ dimensions) Wardidentities, as long as all operators are inserted at separate points.

The Osborn Equation states that the Weyl variation of $W$ gives the Weyl anomalies

$$
\delta_{\sigma} W=\int d^{d} x \sqrt{g} \sigma(x) \mathcal{A}\left(\left\{J_{i}\right\}, g\right)
$$

\footnotetext{
${ }^{1}$ For further discussions and applications see also [5-7].
} 
where $\mathcal{A}$ is the local anomaly polynomial. The generating functional can be chosen to respect diffeomorphism symmetry. Then there are two cohomological structures underlying the Osborn Equation which are related to the realization of the abelian Weyl symmetry:

1. Since the Weyl transformation should obey

$$
\left[\delta_{\sigma_{1}}, \delta_{\sigma_{2}}\right]=0
$$

the transformation of the sources implied by (1.1)

$$
\begin{aligned}
\delta_{\sigma} g_{\mu \nu} & \equiv 2 \sigma g_{\mu \nu} \\
\delta_{\sigma} J_{i} & \equiv \beta_{i}\left(\left\{J_{j}\right\}, g_{\mu \nu} ; \sigma\right)
\end{aligned}
$$

should obey the integrability condition following from (1.3). While (1.4a) obviously obeys it, for $(1.4 \mathrm{~b})$ we get the non-trivial constraint

$$
\delta_{\sigma_{2}} \beta_{i}\left(\left\{J_{j}\right\}, g_{\mu \nu} ; \sigma_{1}\right)=\delta_{\sigma_{1}} \beta_{i}\left(\left\{J_{j}\right\}, g_{\mu \nu} ; \sigma_{2}\right)
$$

where the second variations are calculated using (1.4).

The integrability conditions have trivial solutions corresponding to local changes of variables of the sources

$$
J_{i}^{\prime}(x) \equiv \gamma_{i}\left(\left\{J_{j}\right\}, g_{\mu \nu}\right)
$$

such that $J_{i}^{\prime}(x)$ transforms just following the dimension, i.e. without a non-trivial beta-function:

$$
\delta_{\sigma} J_{i}^{\prime}(x)=\sigma(x)\left(\Delta_{i}-d\right) J_{i}^{\prime}(x)
$$

The solutions of (1.5) modulo (1.6) define the cohomologically nontrivial betafunctions.

Once these non-trivial beta-functions are found, the Weyl anomaly, i.e. the r.h.s. of eq.(1.2), is the solution of a second cohomology problem:

2. The anomaly should obey

$$
\delta_{\sigma_{2}} \int d^{d} x \sqrt{g} \sigma_{1}(x) \mathcal{A}\left(\left\{J_{i}\right\}, g_{\mu \nu}\right)=\delta_{\sigma_{1}} \int d^{d} x \sqrt{g} \sigma_{2}(x) \mathcal{A}\left(\left\{J_{i}\right\}, g_{\mu \nu}\right)
$$

where the variations are calculated using (1.4a) and (1.4b). This is the WessZumino (WZ) consistency condition. Cohomologically nontrivial Weyl anomalies are solutions of (1.8) modulo variations of local diffeo-invariant functionals of $\left\{J_{i}\right\}$ and $g_{\mu \nu}$.

When the beta-function reduces to the classical term (1.7), the Osborn Equation produces the ordinary Weyl anomalies related to single logarithms in the generating functional. The universal information contained in the beta-function is the coefficient of the 
single logarithm in the correlator corresponding to each power of the source in the expansion. The presence of the nontrivial part of the beta-function is related to multiple logarithms in the generating functional: due to the recursive relations incorporated into the equation (1.2) the logarithms in the left hand side of the equation are cancelled producing at the end the anomaly.

The role of the beta-function can be made explicit in the lowest non-trivial order. Consider the correlator of three integer dimensional operators $\mathcal{O}_{i}, \mathcal{O}_{j}, \mathcal{O}_{k}$ which has a double logarithm corresponding to a nonvanishing structure constant (the simplest realization of this situation is for three marginal but not truly marginal operators). This means that at least for one choice of the indices the OPE (operator product expansion) of two operators contains the third one with a logarithmic singularity:

$$
\mathcal{O}_{i}(x) \mathcal{O}_{j}(y)=c_{i j k}|x-y|^{\Delta_{k}-\Delta_{i}-\Delta_{j}} \mathcal{O}_{k}(y)
$$

Together with the logarithm in the $\left\langle\mathcal{O}_{k}(y) \mathcal{O}_{k}(z)\right\rangle$ correlator this produces the double logarithm in the three point function or equivalently in the appropriate term in the generating functional: ${ }^{2}$

$$
W=\ldots+c_{i j k} J_{i} J_{j}\left(\log \left(\square / \mu^{2}\right)\right)^{2} J_{k}+. .+J_{k} \log \left(\square / \mu^{2}\right) J_{k}+\ldots
$$

where we made explicit also the contribution of the $\mathcal{O}_{k}$ two point function and we left out the powers of $\square$ implied by the dimensions of the operators. The singularity in the OPE is translated into the first nontrivial term in the beta-function of the source $J_{k}$ :

$$
\delta_{\sigma} J_{k} \equiv \beta_{k}=\sigma c_{i j k} J_{i} J_{k}+\ldots
$$

Now we can follow the cancellation of the single logarithm in (1.2) corresponding to (1.10): the action of the Weyl variation of the metric on the covariant Laplacian in the first term cancels the variation of the second term when acted upon by $\beta_{k} \frac{\delta}{\delta J_{k}}$.

The recursion relations which are underlying the perturbative validity of the Osborn Equation are a consequence of the analytic structure of the CFT: in momentum space the correlators obey (subtracted) dispersion relations and a finite number of subtractions depending on the dimensions of the operators control all correlators. This is of course valid provided the operators are relevant $\left(\Delta_{i}<d\right)$ or marginal $\left(\Delta_{i}=d\right)$.

For integer dimension irrelevant operators $\left(\Delta_{i}>d\right)$ the number of subtractions needed increases with the order of the correlator, but we expect that for any finite number of irrelevant operators the general structure of the Osborn Equation will be still valid since one needs a finite number of subtractions to control the logarithms.

We will see, however, that in the presence of irrelevant operators a rather basic feature of eq.(1.1) is changed: we have to introduce a non-trivial beta-function also for the transformation of $g_{\mu \nu}$. This changes the definition of the Weyl transformations but it is necessary since correlators of irrelevant operators with energy-momentum tensors acquire

\footnotetext{
${ }^{2}$ the exact position of the double logarithms is not essential for the following argument.
} 
an increasing number of logarithms. This feature becomes evident already for the terms in the generating functional involving only two irrelevant operators when as for relevant or marginal operators the beta-function does not contribute. The sources transform just following their dimension and the Osborn Equation should produce a unique local Weyl anomaly. There is, however, a general proof that such a local expression with the required cohomological properties cannot exist for two irrelevant operators. We correlate this feature with the existence of multiple logarithms in correlators involving energy-momentum tensors and we argue that a "metric beta-function" should be introduced, which modifies the transformation rule of the metric under a Weyl transformation. The non-classical contribution contains information about logarithms and acts on the anomalous part of the effective action, in analogy to the beta-function of the scalar operators which we have discussed before. We analyze in detail the consistency of this assumption in lowest non-trivial order through the OPE involving the energy-momentum tensor and through a general cohomological analysis. We discuss the physical consequences of this generalization, in particular the implications for the RG transformation properties of the geometry dependence when the CFT is formulated on a compact manifold.

In Section 2 we review the Weyl anomaly involving two relevant or marginal operators and the mathematical results determining its form. Similar expressions for two irrelevant operators are proven not to exist. We study the OPE involving the energy-momentum tensor and two irrelevant operators generating a metric beta-function to lowest non-trivial order.

In Section 3 we examine in detail the cohomological structure of the metric beta-function and corresponding anomaly in $d=4$. The explicit expression is worked out for the lowest irrelevant dimension, i.e. $\Delta=5$.

In Section 4 we discuss the peculiar features produced by irrelevant operators in $d=2$, in particular the interplay with type A Weyl anomalies.

In the Conclusions we discuss the general structure of Weyl anomalies involving an arbitrary maximal number of irrelevant operators and energy-momentum tensors. We also point out the implications for the dependence of the partition function on the metric when the theory is formulated on a compact manifold: in the presence of an irrelevant deformation the background geometry is "running" under a RG transformation. The relevance of the irrelevant operator anomalies for the structure of the chiral ring in supersymmetric gauge theories is indicated.

\section{Anomalies and beta-functions}

Given a CFT in $d$ dimensions, consider the two-point function of scalar primary operators of dimension $\Delta$. With a suitable normalization, conformal invariance fixes it to be

$$
\langle\mathcal{O}(x) \mathcal{O}(0)\rangle=\frac{N}{|x|^{2 \Delta}}
$$


For $\Delta-d / 2=n \in \mathbb{N}_{0}$ this is singular, i.e. it has no Fourier transform. It needs regularization and this necessarily introduces a scale. This is most easily done in momentum space where one finds

$$
\langle\mathcal{O}(p) \mathcal{O}(-p)\rangle=(-1)^{n+1} \frac{N \pi^{d / 2}}{2^{2 n} \Gamma(n+1) \Gamma\left(n+\frac{d}{2}\right)} p^{2 n}\left(\log \left(p^{2} / \mu^{2}\right)+c_{n, d}\right)
$$

where the constants $c_{n, d}$ are scheme dependent; they change if one rescales $\mu$. The explicit scale dependence signals a type B conformal anomaly: the two-point function transforms inhomogeneously under dilatations, i.e. rescaling of the coordinates $x^{\mu} \rightarrow e^{\lambda} x^{\mu}$ or, under rescaling the momenta $p_{\mu} \rightarrow e^{-\lambda} p_{\mu}$. Equivalently

$$
\mu \frac{d}{d \mu}\langle\mathcal{O}(p) \mathcal{O}(-p)\rangle=(-1)^{n} \frac{N \pi^{d / 2}}{2^{2 n-1} \Gamma(n+1) \Gamma\left(n+\frac{d}{2}\right)} p^{2 n}
$$

In position space this translates into

$$
\mu \frac{d}{d \mu}\langle\mathcal{O}(x) \mathcal{O}(0)\rangle \propto \square^{n} \delta(x)
$$

Another way to express the anomaly, which was already used in the Introduction, is based on the fact that Weyl invariance in curved space-time implies conformal invariance in Minkowski space. One introduces space-time dependent sources for $\mathcal{O}$ and the energymomentum tensor, $J(x)$ and $g_{\mu \nu}(x)$. The correlation function (2.2) then translates into a term in the generating functional $W$ proportional to

$$
\int d^{d} x \sqrt{g(x)} J(x) \square^{n} \log \left(\square / \mu^{2}\right) J(x)
$$

and the anomaly as the non-invariance of $W$ under Weyl rescaling the sources, i.e.

$$
\delta_{\sigma} W=\int d^{d} x \sigma J \square^{n} J
$$

We have dropped terms which vanish in flat space-time. In this basic form the anomaly expresses a violation of the Ward identity which relates the correlator $\left\langle T_{\mu \nu} \mathcal{O O}\right\rangle$ to the two point function $\langle\mathcal{O O}\rangle$.

Due to the nonabelian nature of the diffeomorphism group the Ward identity is extended to correlators with any number of energy-momentum tensors and the anomaly expression contains metric dependent terms constrained by diffeomorphism invariance. In the general framework of the Osborn Equation this corresponds to the term in the expansion of the effective action which is quadratic in the sources. To this quadratic order the non-trivial part of the beta-function of the sources cannot contribute. The construction of these terms, which depend on the space-time curvature, is well known in the case of relevant and marginal operators, i.e. for $\Delta \leq d$. Of course we assume $\Delta-\frac{d}{2}=0,1,2 \ldots$ as otherwise there is no anomaly. We will now review this case. We start with a marginal operator and its source $J . J$ is invariant under Weyl rescaling and the anomaly is

$$
\delta_{\sigma} W=\int d^{d} x \sqrt{g} \sigma J \Delta_{c} J
$$


where in $d=2, \Delta_{c}=\square$, and in $d=4$,

$$
\Delta_{c}=\square^{2}+2 \nabla_{\mu}\left(R^{\mu \nu}-\frac{1}{3} g^{\mu \nu} R\right) \nabla_{\nu}
$$

Here $\square$ is the covariant Laplacian and $R$ the curvature. They transform as $\Delta_{c} \rightarrow e^{-d \sigma} \Delta_{c}$. In $d=4, \Delta_{c}$ is known as the Fradkin-Tseytlin-Riegert operator in the physics literature and as the (critical) Paneitz operator in the mathematics literature. Higher dimensional generalizations have been constructed; see [8] for a review. The above can be generalized to an arbitrary number of marginal operators and their sources $J^{I}$ in such a way that the anomaly is invariant under reparametrizations of the conformal manifold whose local coordinates are $J^{I}$. For explicit results in $d=2$ and $d=4$, see e.g. [9].

Relevant operators are also easily incorporated. For instance, the source of a dimension two operator in $d=4$ transforms as $J \rightarrow e^{-2 \sigma} J$ and one has $\Delta_{c}=1$, while the source of a dimension three operator in $d=4$ transforms as $J \rightarrow e^{-\sigma} J$. In this case one finds $\Delta_{c}=\square-\frac{1}{6} R$ which transforms as $\Delta_{c} \rightarrow e^{-3 \sigma} \Delta_{c} e^{\sigma}$ under Weyl rescaling of the metric.

We now turn to irrelevant operators, i.e. $\Delta>d$. From the structure of (2.5) we conclude that the Weyl variation of the generating functional should contain a term (2.6) with $n>d / 2$ and the full expression in curved space-time should be Weyl invariant. Assuming that the sources transform homogeneously according to their dimension, i.e.

$$
\delta_{\sigma} g_{\mu \nu}=2 \sigma g_{\mu \nu}, \quad \text { and } \quad \delta_{\sigma} J=(\Delta-d) \sigma J
$$

this would require the existence of an operator

$$
\Delta_{c}=\square^{n}+\text { curvature terms }
$$

such that

$$
\Delta_{c}\left(e^{2 \sigma} g\right)=e^{-\Delta \sigma} \Delta_{c}(g) e^{-(\Delta-d) \sigma}
$$

where, as before, $n=\Delta-d / 2$.

The construction of Weyl anomalies for irrelevant vs. relevant and marginal operators is, however, quite different. This is made evident by a theorem of Gover and Hirachi which excludes the existence of local operators with the simple transformation (2.11). More explicitly, it states (see e.g. [8] for background, review and references to the original literature):

On any (pseudo-)Riemannian manifold $(M, g)$ of dimension d there exists a differential operator of the form

$$
\Delta_{c}=\square^{n}+\text { lower order terms }
$$

such that

$$
\Delta_{c}\left(e^{2 \sigma} g\right)=e^{-\left(\frac{d}{2}+n\right) \sigma} \Delta_{c}(g) e^{+\left(\frac{d}{2}-n\right) \sigma}
$$

with the restriction $1 \leq n \leq \frac{d}{2}$ for even $d$ and no restriction for odd $d$.

This operator is natural in the sense that it is constructed from the metric and the lower order terms vanish in flat backgrounds. Its construction is essentially holographic. 
In general the operator is not unique. For instance, for $n=d / 2=3$ there is a twoparameter family [10]. As there is no issue in odd dimensions, we will restrict to even $d$ in the following and, more specifically, to $d=2$ and $d=4$ and to integer $\Delta \geq d$.

The theorem explicitly excludes the existence of such an operator for $n>d / 2$. Since a diffeomorphism invariant generating functional, whose anomaly reduces to $J \square^{n} J$ in a flat metric should exist, and the analytic structure underlying the Osborn Equation is valid for any finite number of terms in an expansion in the sources of irrelevant operators, there has to be a way to avoid the conclusions of the theorem. This obviously requires a modification in the structure of the equation. In view of the above theorem, the existence of beta-functions for the sources $J^{i}$, including those induced by the irrelevant operators, is not sufficient to lead to a consistent equation. The novel feature, which is only possible in the presence of sources for irrelevant operators ${ }^{3}$, is that the Weyl-transformation of the metric also receives corrections. This is a consequence of logarithms in correlation functions of the energy momentum tensor with irrelevant operators, in much the same way as the beta-functions for the scalar sources arise from logarithms in correlation functions of scalar operators.

We will now present the detailed argument for the existence of a beta-function for the metric in the presence of sources of integer dimension irrelevant operators. To this end we analyze the general form of the $\left\langle T_{\mu \nu} \mathcal{O} \mathcal{O}\right\rangle$ three-point function. ${ }^{4}$ It is fixed by the symmetries, i.e. conservation and tracelessness of $T_{\mu \nu}$, and the normalizations of the $\langle\mathcal{O} \mathcal{O}\rangle$ and $\left\langle T_{\mu \nu} T_{\rho \sigma}\right\rangle$ two-point functions. Comparing it to the latter, which is also completely fixed by the symmetries except for its normalization $c_{T}$, one can extract the following terms in the $\mathcal{O O}$ OPE

$$
\begin{aligned}
\mathcal{O}(x) \mathcal{O}(0) \sim & \frac{N}{x^{2 \Delta}}+\cdots+\frac{a}{c_{T}} \frac{1}{x^{2 \Delta+2-d}} T_{\alpha \beta}(0) x^{\alpha} x^{\beta}+\frac{a}{2 c_{T}} \frac{1}{x^{2 \Delta+2-d}} \partial_{\gamma} T_{\alpha \beta}(0) x^{\alpha} x^{\beta} x^{\gamma} \\
& +\frac{a}{8(d+3) c_{T}} \frac{1}{x^{2 \Delta+2-d}}\left((d+4) \partial_{\gamma} \partial_{\delta} T_{\alpha \beta}(0) x^{\gamma} x^{\delta} x^{\alpha} x^{\beta}-\square T_{\alpha \beta}(0) x^{2} x^{\alpha} x^{\beta}\right)+\ldots \\
& +\frac{1}{x^{2 \Delta-\tilde{\Delta}+n}} \tilde{\mathcal{O}}_{\alpha_{1} \ldots \alpha_{n}} x^{\alpha_{1}} \cdots x^{\alpha_{n}}(0)+\ldots
\end{aligned}
$$

Here

$$
a=-\frac{d \Delta N}{S_{d}(d-1)} \quad\langle\mathcal{O}(x) \mathcal{O}(0)\rangle=\frac{N}{x^{2 \Delta}} \quad S_{d}=\operatorname{Vol}\left(S^{d-1}\right)=\frac{2 \pi^{d / 2}}{\Gamma(d / 2)}
$$

In $d=4$, in the normalization of the type B Weyl anomaly as $g^{\mu \nu}\left\langle T_{\mu \nu}\right\rangle=c C^{2}\left(C_{\mu \nu \rho \sigma}\right.$

\footnotetext{
${ }^{3}$ Simple dimensional arguments and locality immediately imply that a non-trivial beta-function for the metric can only occur in the presence of sources for irrelevant operators. For marginal operators with sources $J$ the only possible modification is $\delta g_{\mu \nu}=2 \sigma f(J) g_{\mu \nu}$.

${ }^{4} \mathrm{~A}$ comprehensive analysis of this and other CFT three-point functions in momentum space was performed in [11]. The singularity structure, which contains all the information relevant for us, can, however, be easily extracted without knowing the full correlation function. We use the results of [12], whose notation we also adopt.
} 
being the Weyl tensor) one finds [13]

$$
c_{T}=-\frac{640}{\pi^{2}} c
$$

The terms in the last line of (2.14) are generic representatives of other primaries (and their descendants). They will contribute to correlation functions such as $\left\langle T_{\mu \nu} T_{\rho \sigma} \mathcal{O} \mathcal{O}\right\rangle$. This will play a role in $d=2$ which we discuss in Section 4. Quite generally, if, suppressing possible tensor structures of the operators,

$$
\mathcal{O}_{j}(x) \mathcal{O}_{k}(0)=\frac{c_{j k}^{l}}{|x|^{\Delta_{j}+\Delta_{k}-\Delta_{l}}} \mathcal{O}_{l}(0)+\ldots \quad \text { with } \quad \Delta_{j}+\Delta_{k}-\Delta_{l}=d+2 n
$$

then there is a beta function for the source of $\mathcal{O}_{l}, \beta^{l}(x) \sim c_{j k}^{l} K^{(n)}\left(J^{j} J^{k}\right)(x)$ where $K^{(n)}$ is an $n$-th order differential operator which acts on the sources, therefore it vanishes for constant sources if $n>0$. The above discussion does not yet take into account special properties such as conservation and tracelessness of e.g. $\mathcal{O}_{l}=T_{\alpha \beta}$. They imply that terms in the beta-function for the metric proportional to the metric itself cannot be obtained in this way.

Using the OPE (2.14) one finds (the derivatives act on $T(0)$ )

$$
\begin{aligned}
& \left\langle T_{\mu \nu}(x) \mathcal{O}(y) \mathcal{O}(0)\right\rangle=\frac{a}{c_{T}} \frac{y^{\alpha} y^{\beta}}{y^{2 \Delta+2-d}}\left\langle T_{\mu \nu}(x) T_{\alpha \beta}(0)+\frac{a}{2 c_{T}} \frac{y^{\alpha} y^{\beta} y^{\gamma}}{y^{2 \Delta+2-d}} \partial_{\gamma}\left\langle T_{\mu \nu}(x) T_{\alpha \beta}(0)\right\rangle\right. \\
& +\frac{a}{8(d+3) c_{T}} \frac{1}{y^{2 \Delta+2-d}}\left((d+4) y^{\alpha} y^{\beta} y^{\gamma} y^{\delta} \partial_{\gamma} \partial_{\delta}-y^{2} y^{\alpha} y^{\beta} \square\right)\left\langle T_{\mu \nu}(x) T_{\alpha \beta}(0)\right\rangle+\ldots
\end{aligned}
$$

The first few terms on the r.h.s., their number depending on $d$ and $\Delta$, need regularization. This introduces a scale $\mu$ and renders $\mu \frac{d}{d \mu}$ of this correlator non-zero. If one takes into account tracelessness and conservation of $T_{\alpha \beta}$, one finds that the terms with up to $n$ derivatives contribute, where $2 \Delta+2-2 d=2(n+2)$. This is easily shown in momentum space. E.g. for $d=4$ and $\Delta=5$, which will be analyzed in detail below, only the first term needs to be considered. This leads to a beta-function for the source of $T_{\alpha \beta}$ if $\Delta>d$, as can also be seen by simple dimensional analysis. This also reveals that the beta-function for the metric vanishes for constant sources.

For $\Delta>d$, the three-point function $\left\langle T_{\mu \nu} \mathcal{O O}\right\rangle$ has a double log in even dimensions $>2$. This follows from the second term in (2.14). For $\Delta=d$ the coefficient of $T_{\alpha \beta}$ is singular and regularization introduces a scale $\mu$. The derivative w.r.t. this scale vanishes because of tracelessness on $T$. For $\Delta>d$ it is proportional to $\partial^{\alpha} \partial^{\beta} \square^{\Delta-d-1} \delta^{(d)}(y)$. In even $d>2$ the two-point function $\left\langle T_{\mu \nu} T_{\alpha \beta}\right\rangle$ provides an additional $\log$. In $d=2$ this two-point function does not have a $\log$ but a $1 / p^{2}$ singularity in momentum space. One can explicitly verify the appearance of $\left(\log \Lambda^{2}\right)^{2}$ terms in $\left\langle T_{\mu \nu} \phi^{n} \phi^{n}\right\rangle$ for the free scalar in $d=4$ if $n \geq 5$.

On the 1.h.s. of the Osborn Equation the logarithms will be cancelled exactly due to the presence of the metric beta-function. The general structure is therefore that in 
correlators of irrelevant operators with energy-momentum tensors, multiple logarithms appear and this necessitates the existence of a metric beta-function such that the l.h.s. of the equation is a local expression, the Weyl anomaly. We propose therefore that in the presence of irrelevant operators with sources $\bar{J}$ the operator of (1.1) is modified to:

$$
\delta_{\sigma} W=\int d^{d} x\left\{\beta_{\mu \nu}^{g}\left(\bar{J}, g_{\mu \nu} ; \sigma\right) \frac{\delta}{\delta g_{\mu \nu}(x)}+\sum_{i} \beta_{i}\left(\left\{J_{j}\right\}, g_{\mu \nu} ; \sigma\right) \frac{\delta}{\delta J_{i}(x)}\right\} W
$$

where $\beta_{\mu \nu}^{g}$ is the metric beta-function. It contains, in addition to the dimensional term $2 \sigma(x) g_{\mu \nu}(x)$, contributions which involve the sources for the irrelevant operators. The second sum is over all scalar sources.

We may also consider $\left\langle T_{\mu \nu}\left(x_{1}\right) T_{\rho \sigma}\left(x_{2}\right) \mathcal{O}\left(x_{3}\right)\right\rangle$. Again, tracelessness and conservation fixes it up to one overall constant [12]. In $d=2$ these conditions imply that it vanishes except if $\Delta=0$ which, in a unitary theory, means $\mathcal{O}=\mathbb{1}$ or if $\Delta=4$, which means $\mathcal{O}=T^{\mu \nu} T_{\mu \nu}$. Expressed in a chiral basis this can be recognized as Zamolodchikov's $T \bar{T}$ [14]. For general $d$, the singularity at $x_{1} \rightarrow x_{2}$ is $x_{12}^{\Delta-2 d}$, which requires regularization for even $\Delta>d$. On the other hand, when in addition also $x_{2} \rightarrow x_{3}$, the singularity is $x_{23}^{-2 \Delta}$. This leads to an anomaly linear in the source and quadratic in $h_{\mu \nu}$, where $g_{\mu \nu}=\eta_{\mu \nu}+h_{\mu \nu}$.

The question of energy-momentum correlators which contain multiple logarithms is a very interesting one and in general depends on the specific structure of the CFT. In the present paper we will mainly discuss the multiple logarithms generated by the interplay of the $\langle\mathcal{O O}\rangle,\langle T \mathcal{O O}\rangle$ and $\langle T T\rangle$ correlators. They exist in any CFT once the integer dimensional irrelevant operator $\mathcal{O}$ is present.

\section{$3 \mathrm{~d}=4$}

In this section we make the above discussion concrete and derive a non-zero beta function for the metric by combining the regularized correlation function $\left\langle T_{\mu \nu} \mathcal{O} \mathcal{O}\right\rangle$ with the Osborn Equation. ${ }^{5}$ A concrete realization, on which the general arguments can be explicitly checked, is a free scalar field $\phi$ with $\phi^{n}$ being an operator of dimension $\Delta=n$.

We start by including the lowest integer dimension irrelevant operator, which has dimension $\Delta=5$. We denote its source by $\rho$. Some terms in the generating functional, which will play a role in the following discussion, are

$$
\begin{aligned}
W[\rho, h]=\frac{1}{2} \int d^{4} x d^{4} y\langle\mathcal{O}(x) \mathcal{O}(y)\rangle \rho(x) \rho(y)+\frac{1}{2} \int d^{4} x d^{4} y\left\langle T_{\mu \nu}(x) T_{\alpha \beta}(y)\right\rangle h^{\mu \nu}(x) h^{\alpha \beta}(y) \\
+\frac{1}{2} \int d^{4} x d^{4} y d^{4} z\left\langle T_{\mu \nu}(x) \mathcal{O}(y) \mathcal{O}(z)\right\rangle h^{\mu \nu}(x) \rho(y) \rho(z)+\ldots
\end{aligned}
$$

\footnotetext{
${ }^{5}$ This extends the analysis of [15] where only scalar operators were considered and the extension to a curved background metric was not addressed.
} 
All correlation functions are computed in the original CFT. These terms are present in any CFT which has a dimension five operator. The first and third terms in (3.1) should be considered together since they are related by the diffeomorphism Ward identity which we will always implement.

For $d=4$ and $\Delta=5(2.18)$ becomes (the derivatives are w.r.t. the argument of $T_{\alpha \beta}$ )

$$
\begin{aligned}
\left\langle T_{\mu \nu}(x) \mathcal{O}(y) \mathcal{O}(0)\right\rangle & =\frac{a}{c_{T}} \frac{y^{\alpha} y^{\beta}}{y^{8}}\left\langle T_{\mu \nu}(x) T_{\alpha \beta}(0)+\frac{a}{2 c_{T}} \frac{y^{\alpha} y^{\beta} y^{\gamma}}{y^{8}} \partial_{\gamma}\left\langle T_{\mu \nu}(x) T_{\alpha \beta}(0)\right\rangle\right. \\
& +\frac{a}{56 c_{T}}\left(8 \frac{y^{\alpha} y^{\beta} y^{\gamma} y^{\delta}}{y^{8}} \partial_{\gamma} \partial_{\delta}-\frac{y^{\alpha} y^{\beta}}{y^{6}} \square\right)\left\langle T_{\mu \nu}(x) T_{\alpha \beta}(0)\right\rangle+\ldots
\end{aligned}
$$

We have only displayed those terms which require regularization, which introduces a renormalization scale and hence type B anomalies and beta-functions. Due to tracelessness and conservation of $T_{\alpha \beta}$ only the first term contributes and one finds

$$
\mu \frac{d}{d \mu}\left\langle T_{\mu \nu}(x) \mathcal{O}(y) \mathcal{O}(0)\right\rangle=\frac{a \pi^{2}}{24 c_{T}} \partial^{\alpha} \partial^{\beta} \delta^{(4)}(y)\left\langle T_{\mu \nu}(x) T_{\alpha \beta}(0)\right\rangle
$$

This can be derived either in position space using differential regularization [16] or, more straightforwardly, in momentum space. Therefore

$$
\begin{aligned}
\mu \frac{d}{d \mu} W & =\frac{a \pi^{2}}{24 c_{T}} \int d^{4} x d^{4} z\left\langle T_{\mu \nu}(x) T_{\alpha \beta}(z)\right\rangle h^{\mu v}(x) \rho(z) \partial^{\alpha} \partial^{\beta} \rho(z)+\ldots \\
& =\frac{a \pi^{2}}{24 c_{T}} \int d^{4} z \rho(z) \partial_{\alpha} \partial_{\beta} \rho(z) \frac{\delta}{\delta h^{\alpha \beta}(z)} W+\ldots
\end{aligned}
$$

We require that $W$ satisfies the Osborn Equation (1.2), i.e. after acting with the Weyl variation operator the terms with single logarithms are cancelled. This cancellation is between a contribution of the third term (a variation of a double logarithm) and a contribution of the second term which needs a $\mathcal{O}\left(\rho^{2}\right)$ contribution to the Weyl variation of the metric

$$
\delta_{\sigma} g_{\mu \nu}=2 \sigma g_{\mu \nu}+\frac{a \pi^{2}}{24 c_{T}} \sigma \rho \partial_{\mu} \partial_{\nu} \rho+\ldots
$$

Here ... are possible terms contributing to $\beta_{\mu \nu}^{g}$ that cannot be derived by the above analysis, which is based on the two- and three-point functions. These are terms which either vanish in flat space or for constant $\sigma$, terms which are proportional to $g_{\mu \nu}$ and, of course, terms of higher order in $\rho$. Once the logarithms in the variation are cancelled, there is a local anomaly which to this order - second order in $\rho$ and zeroth order in the metric - comes from the first term in (3.1) and it is $\sim \rho \square^{3} \rho$.

We will now determine all terms to $\mathcal{O}\left(\rho^{2}\right)$, but to all orders in the metric, seeded by the lowest terms (3.1) by performing a cohomological analysis. Once this is achieved, we determine the anomaly by imposing WZ consistency.

To find the complete beta-function for the metric to $\mathcal{O}\left(\rho^{2}\right)$ or, equivalently, to second order in derivatives, we start with the most general Ansatz for $\delta_{\sigma} g_{\mu \nu}$ to this order, which 
is consistent with dimensional analysis. We then extend and perform the cohomological analysis outlined in the Introduction for $\beta_{\mu \nu}^{g}(\rho, g, \sigma)$, i.e. we impose $\left[\delta_{\sigma_{1}}, \delta_{\sigma_{2}}\right] g_{\mu \nu}=0$ which leads to (1.5) for $\beta_{\mu \nu}^{g}$. This is solved modulo trivial solutions, i.e. local expressions $\gamma_{\mu \nu}$ s.t. such that $\delta_{\sigma}\left(g_{\mu \nu}+\gamma_{\mu \nu}\right)=2 \sigma\left(g_{\mu \nu}+\gamma_{\mu \nu}\right)$.

For the transformation of $\rho$ we have to this order

$$
\delta_{\sigma} \rho=\sigma \rho
$$

For the metric the general Ansatz is

$$
\begin{aligned}
\delta_{\sigma} g_{\mu \nu} & =2 \sigma g_{\mu \nu}+c_{1} \sigma \nabla_{\mu} \rho \nabla_{\nu} \rho+c_{2} \rho^{2} \nabla_{\mu} \nabla_{\nu} \sigma+c_{3} \sigma \rho \nabla_{\mu} \nabla_{\nu} \rho+c_{4} \rho\left(\nabla_{\mu} \rho \nabla_{\nu} \sigma+\nabla_{\nu} \rho \nabla_{\mu} \sigma\right) \\
& +c_{5} \sigma g_{\mu \nu} \rho \square \rho+c_{6} \sigma g_{\mu \nu} \nabla^{\alpha} \rho \nabla_{\alpha} \rho+c_{7} \sigma g_{\mu \nu} \rho^{2} R+c_{8} g_{\mu \nu} \rho^{2} \square \sigma \\
& +c_{9} g_{\mu \nu} \rho \nabla^{\alpha} \rho \nabla_{\alpha} \sigma+c_{10} \sigma R_{\mu \nu} \rho^{2}
\end{aligned}
$$

Imposing consistency (1.5) leaves, in general dimension, six solutions: the ones parametrized by $c_{2}, c_{4}, c_{8}$ and $c_{9}$, which can be shown to be cohomologically trivial in the sense described above;

$$
\sigma\left(R_{\mu \nu} \rho^{2}+(d-2) \rho \nabla_{\mu} \nabla_{\nu} \rho-(d-1) g_{\mu \nu}(\nabla \rho)^{2}+g_{\mu \nu} \rho \square \rho\right) \equiv \sigma \rho^{2} \hat{R}_{\mu \nu}
$$

and

$$
g_{\mu \nu} \sigma\left(R \rho^{2}+2(d-1) \rho \square \rho-d(d-1)(\nabla \rho)^{2}\right) \equiv \sigma \rho^{2} \hat{g}_{\mu \nu} \hat{R}
$$

Here $\hat{R}$ is the curvature computed with the Weyl-invariant metric $\hat{g}_{\mu \nu}=\frac{1}{\rho^{2}} g_{\mu \nu}$. We choose the first solution in (3.8). It contains the "seed" (3.5) and extends it to all orders in $g_{\mu \nu}$. Adding (3.9) would not modify the result which we are going to find for the anomaly. A general discussion of beta-functions with the structure of the second solution, i.e. nontrivial consistent solution proportional to $g_{\mu \nu}$, multiplied by a Weyl invariant structure, will be given later.

We now make the general Ansatz for the anomaly $\mathcal{A}\left(\rho, g_{\mu \nu}\right)$ to $\mathcal{O}\left(\rho^{2}\right)$ or, equivalently, to sixth order in derivatives, and require that it satisfies the WZ consistency condition (1.8). We use the normalization

$$
\delta_{\sigma} g_{\mu \nu}=2 \sigma g_{\mu \nu}+\alpha \sigma \rho^{2} \hat{R}_{\mu \nu}
$$

where $\alpha$ is related to $a$ in (2.18), i.e. to the normalization of $\left\langle T_{\mu \nu} \mathcal{O} \mathcal{O}\right\rangle$, as will be made explicit below. One then finds the following solution of the WZ consistency condition

$$
\begin{aligned}
& \mathcal{A}=c C_{\mu \nu \rho \sigma} C^{\mu \nu \rho \sigma}+\alpha c\left\{\square \rho \square^{2} \rho-\frac{13}{8} R R^{\mu \nu} R_{\mu \nu} \rho^{2}+\frac{53}{162} R^{3} \rho^{2}+\frac{4}{3} R^{\mu \nu} R^{\rho \sigma} R_{\mu \rho \nu \sigma} \rho^{2}\right. \\
& -\frac{1}{8} R R_{\mu \nu \rho \sigma} R^{\mu \nu \rho \sigma} \rho^{2}+\frac{43}{72} R_{\mu \nu \rho \sigma} R^{\mu \nu \alpha \beta} R_{\alpha \beta}^{\rho \sigma} \rho^{2}-\frac{35}{72} R^{2} \rho \square \rho+\frac{25}{24} R_{\mu \nu \rho \sigma} R^{\mu \nu \rho \sigma} \rho \square \rho \\
& -\frac{1}{36} \nabla^{\mu} R \nabla_{\mu} R \rho^{2}+\frac{167}{12} R^{\mu \nu} R_{\mu \nu} \nabla^{\alpha} \rho \nabla_{\alpha} \rho-\frac{101}{24} R^{2} \nabla^{\alpha} \rho \nabla_{\alpha} \rho
\end{aligned}
$$




$$
\begin{aligned}
& -\frac{79}{24} R^{\mu \nu \rho \sigma} R_{\mu \nu \rho \sigma} \nabla^{\alpha} \rho \nabla_{\alpha} \rho-\frac{1}{3} R \square \nabla^{\mu} \rho \nabla_{\mu} \rho-\frac{10}{9} R^{\mu \nu} \nabla_{\mu} \nabla_{\nu} R \rho^{2}+\frac{7}{9} R^{\mu \nu} R \rho \nabla_{\mu} \nabla_{\nu} \rho \\
& +\frac{1}{36} \square R \rho \square \rho-\frac{16}{9} R(\square \rho)^{2}+\nabla^{\mu} R \nabla_{\mu} \rho \square \rho+\frac{1}{6} R \rho \square^{2} \rho-4 R^{\mu \nu} \nabla_{\mu} \rho \square \nabla_{\nu} \rho \quad(3.11) \\
& -\frac{37}{18} R_{\mu \nu} \nabla^{\mu} R \rho \nabla_{\nu} \rho-22 R_{\mu}^{\alpha} R_{\nu \alpha} \nabla^{\mu} \rho \nabla^{\nu} \rho+\frac{116}{9} R^{\mu \nu} R \nabla^{\mu} \rho \nabla^{\nu} \rho \\
& -13 R^{\alpha \beta} R_{\mu \alpha \nu \beta} \nabla^{\mu} \rho \nabla^{\nu} \rho-\frac{5}{18} \nabla^{\mu} \nabla^{\nu} R \rho \nabla_{\mu} \nabla_{\nu} \rho-\frac{5}{9} R \nabla_{\mu} \nabla_{\nu} \rho \nabla^{\mu} \nabla^{\nu} \rho \\
& -5 R^{\beta \gamma} \nabla_{\gamma} R_{\alpha \beta} \rho \nabla^{\alpha} \rho-\frac{8}{3} R_{\alpha}^{\gamma} R^{\alpha \beta} \rho \nabla_{\beta} \nabla_{\gamma} \rho+\frac{10}{3} R^{\beta \gamma} \nabla^{\alpha} \rho \nabla_{\gamma} \nabla_{\beta} \nabla_{\alpha} \rho \\
& \left.+\frac{5}{6} \square R^{\mu \nu} \rho \nabla_{\mu} \nabla_{\nu} \rho+\frac{22}{3} R^{\mu \nu} \nabla_{\mu} \nabla_{\nu} \rho \square \rho-\frac{5}{3} \nabla^{\mu} R^{\alpha \beta} \nabla_{\mu} R_{\alpha \beta} \rho^{2}\right\}+\mathcal{O}\left(\rho^{4}\right)
\end{aligned}
$$

This anomaly is entirely type $\mathrm{B}$, as it does not vanish for constant Weyl parameter $\sigma$, i.e. neither the $\mathcal{O}\left(\rho^{0}\right)$ nor the $\mathcal{O}\left(\rho^{2}\right)$ parts of $\mathcal{A}$ are total derivatives. The second term is what we obtained before to lowest order. The remaining terms are higher order in the metric and they vanish in flat space. They implement diffeomorphism invariance. Note the interplay between the $\rho$-independent ordinary type B Weyl anomaly and the $\rho$-dependent terms, which is possible due to the $\rho$-dependence of $\delta_{\sigma} g_{\mu \nu}$, which is in turn required to avoid the conclusions from the theorem of Gover and Hirachi.

Comparing (3.10) with (3.5) we identify

$$
2 \alpha=\frac{a \pi^{2}}{24 c_{T}}=\frac{N \pi^{2}}{3^{2} \cdot 2^{9} c}
$$

The anomaly in the two-point function $\langle\mathcal{O O}\rangle$ derived from the above anomaly polynomial is

$$
2 c \alpha \square^{3} \delta^{(4)}(x)=\frac{N \pi^{2}}{2^{9} \cdot 3^{2}} \square^{3} \delta^{(4)}(x)
$$

which agrees with the (anomalous) change of (2.2) under scale transformation.

By adding a cohomologically trivial term

$$
\Delta W=\frac{\alpha c}{24} \int d^{4} x \sqrt{g}\left(6 \square \rho \square^{2} \rho-2 R \rho \square^{2} \rho-R(\square \rho)^{2}\right)
$$

one can eliminate the second term in (3.11) in favour of $\rho \square^{3} \rho$; of course the curvature dependent terms will also change.

The anomaly (3.11) can be understood as "seeded" by the correlator of two irrelevant operators. Once the metric beta-function is turned on, the usual forms of the Weyl anomalies (type A and B) are no longer valid since the Weyl variation of the metric has changed. As a consequence the corresponding expressions will be deformed by the metric beta-function. In this sense (3.11) can be understood also as the appropriate deformation of the "c" Weyl anomaly, as the presence of the first term shows. The information 
contained in (3.11) is universal, i.e. independent on the regularization scheme. It is theory dependent through the two parameters which give the coefficients of single logarithms in the correlator of two energy-momentum tensors and two irrelevant operators, respectively.

A similar deformation occurs for the type A Euler anomaly in $d=4$. The cohomologically nontrivial anomaly starting with the Euler density, when the metric variation contains the deformation (3.10), is

$$
\begin{aligned}
\mathcal{A}= & a E_{4}+\alpha a\left\{\frac{28}{135} R^{3} \rho^{2}-\frac{6}{5} R^{\mu \nu} R^{\rho \sigma} R_{\mu \rho \nu \sigma} \rho^{2}-\frac{7}{10} R R^{\mu \nu \rho \sigma} R_{\mu \nu \rho \sigma} \rho^{2}\right. \\
& +\frac{14}{15} R_{\mu \nu \rho \sigma} R^{\rho \sigma \alpha \beta} R_{\alpha \beta}{ }^{\mu \nu} \rho^{2}-\frac{1}{15} R \square R \rho^{2}+\frac{2}{3} R^{2} \rho \square \rho+\frac{58}{5} R^{\mu \nu} R_{\mu \nu}(\nabla \rho)^{2} \\
& -\frac{64}{15} R^{2}(\nabla \rho)^{2}+\frac{1}{5} R^{\mu \nu \rho \sigma} R_{\mu \nu \rho \sigma}(\nabla \rho)^{2}-12 R_{\mu \rho} R_{\nu}{ }^{\rho} \nabla^{\mu} \rho \nabla^{\nu} \rho+8 R R_{\mu \nu} \nabla^{\mu} \rho \nabla^{\nu} \rho \\
& -8 R^{\rho \sigma} R_{\mu \rho \nu \sigma} \nabla^{\mu} \rho \nabla^{\nu} \rho+\frac{2}{5} R^{\mu \nu} \square R_{\mu \nu} \rho^{2}-2 R^{\mu \nu} R_{\mu \nu} \rho \square+\frac{7}{5} \nabla^{\mu} R^{\nu \rho} \nabla_{\nu} R_{\mu \rho} \rho^{2} \\
& \left.-\frac{7}{10} \nabla^{\rho} R^{\mu \nu} \nabla_{\rho} R_{\mu \nu} \rho^{2}-\frac{4}{5} \nabla^{\sigma} \nabla^{\rho} R^{\mu \nu} R_{\mu \rho \nu \sigma} \rho^{2}-\frac{7}{20} \nabla^{\alpha} R^{\mu \nu \rho \sigma} \nabla_{\alpha} R_{\mu \nu \rho \sigma} \rho^{2}\right\}
\end{aligned}
$$

Here

$$
E_{4}=R^{2}-4 R^{\mu \nu} R_{\mu \nu}+R^{\mu \nu \rho \sigma} R_{\mu \nu \rho \sigma}
$$

is the Euler density normalized s.t. $\int_{S^{4}} \sqrt{g} E_{4}=64 \pi^{2}$. This deformed anomaly does not introduce any new universal parameter.

The terms in the Osborn Equation, whose variation is given by (3.15), have joint (single) logarithmic and powerlike ("type A") singularities. They can be understood as originating in a decomposition of correlators of two $\mathcal{O}$ operators with three energy-momentum tensors, where the two irrelevant operators connect through the energy-momentum tensor conformal block.

It might be interesting to note that while (3.11) vanishes in a maximally symmetric space, e.g. $S^{4}$, for constant source $\rho$, this is not the case for (3.15). On the other hand (3.15) vanishes in flat space while (3.11) does not (for non-constant sources).

The deformation of the cohomologically trivial solution $\int d^{4} x \sqrt{g} \sigma \square R$ of the WessZumino condition continues to be trivial, being the variation of the local term $\int d^{4} x \sqrt{g} R^{2}$ under the deformed operator.

To the anomalies determined above, related to the order $\rho^{2}$ contribution in the metric beta-function, we can add "ordinary" anomalies, i.e. type B anomalies with quadratic $\rho$ dependence. If the new structures lead to Weyl invariant anomaly integrands when $g_{\mu \nu}$ transforms naively, these anomalies are also consistent to order $\rho^{2}$ when the metric beta-function is taken into account. There are eight such structures. Two of them are 
simple as they are constructed from the Weyl invariants ${ }^{6}$

$$
\begin{aligned}
I_{1}= & C_{\mu \nu \rho \sigma} C^{\rho \sigma \alpha \beta} C_{\alpha \beta}{ }^{\mu \nu} \\
I_{2}=- & \nabla^{\alpha} R^{\mu \nu \rho \sigma} \nabla_{\alpha} R_{\mu \nu \rho \sigma}-16 R_{\mu \rho \nu \sigma} \nabla^{\sigma} \nabla^{\rho} R^{\mu \nu}-10 \nabla^{\mu} R^{\nu \rho} \nabla_{\mu} R_{\nu \rho}+12 \nabla^{\mu} R^{\rho \sigma} \nabla_{\rho} R_{\mu \sigma} \\
& +8 R^{\mu \nu} \square R_{\mu \nu}+\frac{2}{3} \nabla^{\mu} R \nabla_{\mu} R-\frac{4}{3} R \square R-\frac{2}{3} R R^{\mu \nu \rho \sigma} R_{\mu \nu \rho \sigma}+8 R^{\mu \nu} R^{\rho \sigma} R_{\mu \rho \nu \sigma} \\
& \quad-8 R_{\mu}{ }^{\nu} R_{\nu}{ }^{\rho} R_{\rho}{ }^{\mu}+\frac{4}{3} R R^{\mu \nu} R_{\mu \nu}-\frac{2}{9} R^{3}
\end{aligned}
$$

There is a third simple solution which involves derivatives of $\rho$ :

$$
I_{3}=\hat{R} C_{\mu \nu \alpha \beta} C^{\mu \nu \alpha \beta}
$$

We will not write down the other five solutions which, as $I_{3}$, also contain terms which vanish for constant source $\rho . I_{i}, i=1,2$, transform under Weyl rescaling of the metric as $\delta_{\sigma} I_{i}=-6 \sigma I_{i}$ while $I_{i}, i=3, \ldots, 8$, transform under a joint rescaling of the metric and $\rho$ as $\delta_{\sigma} I_{i}=-4 \sigma I_{i}$. They do not exist in the absence of $\rho$.

The type B anomalies constructed from these structures are $\int d^{4} x \sigma \sqrt{g} \rho^{2} I_{i}$ for $i=1,2$ and $\int d^{4} x \sigma \sqrt{g} I_{i}, i=3, \ldots, 8$, respectively. They correspond to variations of single logarithms in the generating functional. As we will see in the continuation, their appearance could also be related to sources for higher dimension operators. Their coefficients are theory dependent and in particular can vanish in counterdistinction from the anomaly (3.11).

We remark that the anomaly based on $I_{3}$ above can be understood as a deformation of the " $c$ " trace anomaly by (3.9). It is then natural to conjecture that contributions of the type (3.9) to the metric beta-function proportional to $g_{\mu \nu}$ do not represent genuine modifications required by the appearance of multiple logarithms, but simply reproduce ordinary higher order type B anomalies.

We should discuss the stability of these structures when beta-functions for other operators induced by the dimension five operator are also included. If we include only terms up to quadratic order in $\rho$, the only other operators which have to be considered are relevant, marginal and dimension six irrelevant operators. In order that the induced beta-functions contribute in the Osborn Equation we should limit the new couplings to first order. We can then invert the logic and start with contributions of the new operators at linear order in their sources and subsequently study the deformations to order $\rho^{2}$.

We begin by including the source $\tau$ for an operator $\tilde{\mathcal{O}}$ of dimension six. Generically $\langle\mathcal{O} \mathcal{O} \tilde{\mathcal{O}}\rangle \neq 0$, e.g. for a free scalar field with $\mathcal{O}=\phi^{5}$ and $\tilde{\mathcal{O}}=\phi^{6}$. As a consequence there is a beta-function for $\tau$ given by

$$
\delta_{\sigma} \tau=2 \sigma \tau+\beta \sigma \rho^{2}
$$

\footnotetext{
${ }^{6}$ There is a second contraction of three Weyl tensors, but in dimensions $<6$ it is not independent of $I_{1}$.
} 
Here $\beta$ is proportional to the OPE coefficient $c_{\mathcal{O O} \tilde{\mathcal{O}}}$. Therefore the dimension six operator can indeed give contributions to the Osborn Equation to order $\rho^{2}$ and we should study its influence to linear order in $\tau$.

Repeating the cohomological analysis for possible metric beta-functions, one finds that there is no cohomologically nontrivial solution to order $\tau$. As a consequence when the deformation (3.19) is included there will be no modification of (3.8) due to the presence of $\tau$. We can analyze now the Weyl anomalies to order $\tau$. The correlator $\langle T T \tilde{\mathcal{O}}\rangle$ has a logarithmic singularity and we expect therefore that type B anomalies linear in $\tau$ will exist which are at least quadratic in the curvature. Indeed there are four such solutions of the WZ-condition. Clearly $\tau I_{i}$ for $i=1,2$ are solutions. The other two can e.g. be obtained if one replaces $\rho=\sqrt{\tau}$ and requires regularity in $\tau$. This is satisfied by two of the $I_{i}, i=4, \ldots, 8$, say $I_{4}$ and $I_{5}$ :

$$
\delta_{\sigma} W=\int d^{4} x \sqrt{g} \sigma\left\{\tau\left(c_{1} I_{1}(g)+c_{2} I_{2}(g)\right)+c_{4} I_{4}(g, \sqrt{\tau})+c_{5} I_{5}(g, \sqrt{\tau})\right\}
$$

with $I_{1,2}$ as in (3.17). In the presence of the $\rho$-dependent terms in (3.19), the anomaly (3.20) is no longer a consistent solution of the Osborn Equation. The deformation of the anomaly to $\mathcal{O}\left(\rho^{2}\right)$, ignoring terms of order $\tau \rho^{2}$, are precisely the $\int d^{4} x \sqrt{g} \sigma \rho^{2} I_{i}(g), i=1,2$ and $\int d^{4} x \sqrt{g} \sigma I_{i}(g, \rho), i=4,5$ discussed before. These anomalies do not mix with (3.11) and now they acquire a natural interpretation: they originate in the coupling of two irrelevant dimension five operators to the dimension six operator conformal block in the correlator with energy-momentum tensors. Therefore the dimension six operator, even though it has an induced beta-function to order $\rho^{2}$, does not change the structure of the metric beta-function and Weyl anomaly produced by the dimension five operator alone.

We can perform a similar analysis for a marginal (but not necessarily exactly marginal) operator $M$ coupled to a source $J$. We analyze the cohomology problems linear in $J$. The induced beta-function for $J$ is

$$
\delta_{\sigma} J=\gamma \sigma \hat{R}
$$

The coefficient $\gamma$ is proportional to the $c_{M \mathcal{O O}}$ OPE coefficient. This obviously satisfies the consistency condition and is non-trivial. The appearance of $\hat{R}$, or rather its flat space limit, can be verified by studying the $\langle M \mathcal{O O}\rangle$ correlation functions. As follows from our general analysis, a marginal operator can generate in the metric beta-function only a term proportional to the metric itself with a $J$-dependent coefficient function. As argued before such a term cannot contribute to the genuinely new anomaly structure related to multiple logarithms and therefore will not interfere with (3.8). There is a type B anomaly linear in $J$ related to the standard " $c$ " Weyl anomaly

$$
\int d^{4} x \sqrt{g} \sigma J C_{\mu \nu \alpha \beta} C^{\mu \nu \alpha \beta}
$$

After having understood the structure at linear order in $J$, we can deform it using (3.21). The metric beta-function (3.8) will not be changed while the deformation of (3.22) leads to the anomaly based on the $I_{3}$-structure above. We have again a new interpretation of 
this order $\rho^{2}$ anomaly as resulting from a decomposition of the correlator of two irrelevant operators and energy-momentum tensors, where the irrelevant operators couple through the marginal $(J)$ conformal block. We therefore conclude that at $\mathcal{O}\left(\rho^{2}\right)$ there is no change in the metric beta-function (3.8) or the Weyl anomaly (3.11). We do not include a similar analysis for relevant operators. They also receive an induced beta-function at order $\rho^{2}$, the conclusion being the same, i.e. stability of genuine $\rho^{2}$ contribution.

In $d=4$ we have therefore a clear distinction between contributions to the beta-function and anomalies related to multiple logarithms and usual type B contributions in correlators with energy-momentum tensors. We can even characterize the difference by singling out the conformal block through which the irrelevant operators are connected to the energymomentum tensors in a given decomposition scheme. It is an interesting question how this information constraints decompositions related by crossing.

In $d=2$ the stability analysis of the metric beta-function related to induced couplings is much more subtle. This will be discussed in detail the next section.

Let us also stress once more that throughout we worked to order six in derivatives or, equivalently, to order two in $\rho$. There will be contributions at all higher higher orders in $\rho$, but they will only start contributing in correlation functions with more than two insertions of $\mathcal{O}$. At higher orders we will also have to take into account the mixing with operators of dimension $\Delta>5$, namely of those operators which appear in the $\mathcal{O O}$ OPE.

We treated in detail above the structure induced in second order by the minimal integer dimension irrelevant operator. This generalizes to higher dimensional operators but with increasingly complex expressions. We limit ourselves to a discussion of the salient features of the generalization. We consider the anomaly seeded by the quadratic correlator of a dimension six operator whose coupling we continue to denote by $\tau$, but working now to $\mathcal{O}\left(\tau^{2}\right)$. We check first whether there is a non-trivial beta function for the metric. Making the general Ansatz for $\delta g_{\mu \nu}$ at $\mathcal{O}\left(\tau^{2}, \nabla^{6}\right)$, imposing non-triviality and the WZ condition, one finds a five-parameter family of solutions. None of the solutions contains derivatives of $\sigma$. This means that there is no interference between the $\mathcal{O}\left(\tau^{0}\right)$ and the $\mathcal{O}\left(\tau^{2}\right)$ terms and they satisfy the WZ condition independently. Two of the solutions are proportional to $g_{\mu \nu}$ and, as argued above, will not represent "genuine" contributions related to multiple logarithms.

Among the three remaining solutions only one survives in the flat limit and can in principle be extracted from $\langle T \mathcal{O O}\rangle$. In contrast to $\Delta=5$, for $\Delta=6$ more than just the first term on the r.h.s. of (2.2) which contributes to $\langle T \mathcal{O O}\rangle$ needs to be considered. More precisely, as follows from the discussion below eq.(2.18), the second term on the r.h.s. of this equation now also contributes.

One wonders whether these solutions can be constructed, as in case $\Delta=5$ above, from expression constructed from the invariant metric $\hat{g}_{\mu \nu}=\frac{1}{\tau} g_{\mu \nu}$. For instance $\sigma \tau \hat{R} \hat{R}_{\mu \nu}$ transforms homogeneously with weight two, but it is singular in the limit $\tau \rightarrow 0$. There are nine such terms with four derivatives of which five are regular as $\tau \rightarrow 0$. They agree 
with the non-trivial solutions of the WZ condition.

\section{$4 \quad \mathrm{~d}=2$}

The basic structure in $d=2$ is the same as in higher even dimensional theories, i.e. there is a beta-function originating in a logarithmically singular coefficient in front of $T$ in the OPE of two irrelevant operators $\mathcal{O}$. But there are specific $d=2$ features which change the analysis. In particular, in $d=2$ the energy-momentum tensor two-point function has a "type A" anomaly, i.e. the logarithmic dependence is replaced by a singular $1 / \square$ dependence. This will be a general feature, i.e. the multiple logarithms in the various correlators studied will change into one logarithm multiplied by integer power singularities. More generally, even though in the diffeomorphism preserving scheme we are using only the $S O(2,2)$ symmetry is explicit, there is the underlying Virasoro symmetry which implies additional restrictions. We will study again the minimal dimension irrelevant operator which in $d=2$ is $\Delta=3$, its source $\rho$ having the Weyl transformation (3.6).

A concrete realization of such a CFT is a theory of three free Majorana fermions. The dimension three irrelevant operator is

$$
\mathcal{O} \equiv \bar{\psi}_{1} \bar{\psi}_{2} \bar{\psi}_{3} \psi_{1} \psi_{2} \psi_{3}
$$

where $\bar{\psi}, \psi$ are the left and right Majorana fields, respectively .

We will start by solving the cohomological problems in the most general setup to quadratic order in $\rho$. At this order there is an additional "induced" source $\tau$ coupled to a dimension 4 operator $\tilde{O}$. One can then check that the following transformations are non-trivial solutions of the WZ condition:

$$
\begin{aligned}
\delta_{\sigma} \rho & =\sigma \rho \\
\delta_{\sigma} \tau & =2 \sigma \tau+\alpha \sigma \rho^{2} \\
\delta_{\sigma} g_{\mu \nu} & =2 \sigma g_{\mu \nu}+\tau \nabla_{\mu} \nabla_{\nu} \sigma+\alpha \sigma\left(\rho \nabla_{\mu} \nabla_{\nu} \rho-\frac{1}{2} g_{\mu \nu}(\nabla \rho)^{2}\right)
\end{aligned}
$$

The second term in $\delta_{\sigma} \tau$ is the "induced" coupling whose normalization $\alpha$ reflects the $c_{\mathcal{O O} \tilde{\mathcal{O}}}$ structure constant. The coefficient of the second term in $\delta_{\sigma} g_{\mu \nu}$ has been set to one by a suitable rescaling of $\tau$, but it cannot be set to zero. Indeed, in contrast to $d=4$, the metric beta-function now depends on $\tau$ and therefore the mixing between the dimension three and four operators cannot be neglected in the anomaly analysis. The peculiar features of these solutions will be discussed later.

There are contributions to order $\rho^{2}$ also to the marginal and relevant operators but they do not contribute to the anomaly: we will analyse later in this Section their detailed structure and especially the differences compared with the role of $\tau$.

The presence of the metric beta-function once again means that there is an interplay between the purely gravitational part of the Type A anomaly, the Polyakov term $c R$, 
and the part which depends on the sources $\rho$ and $\tau$. The most general Ansatz for the anomaly, after subtracting variations of local terms, can be reduced to

$$
\begin{gathered}
\delta_{\sigma} W=\int \sqrt{g} \sigma\left(c R+a_{1} R^{2} \rho^{2}+a_{2} \rho^{2} \square R+a_{3} R \rho \square \rho+a_{4} R(\nabla \rho)^{2}\right. \\
\left.+a_{5} \rho \square^{2} \rho+a_{6} R^{2} \tau+a_{7} \tau \square R+a_{8} R \square \tau\right)
\end{gathered}
$$

Imposing $\mathrm{WZ}$ on the anomaly leads to the following unique solution

$$
\delta_{\sigma} W=\int \sqrt{g} \sigma c\left(R+\frac{1}{16} \alpha R^{2} \rho^{2}+\frac{1}{4} \alpha R \rho \square \rho-\frac{1}{2} \alpha R(\nabla \rho)^{2}+\frac{1}{4} \alpha \rho \square^{2} \rho\right)
$$

Adding the variation of a local term proportional to $(\square \rho)^{2}$, this becomes

$$
\delta_{\sigma} W=\int \sqrt{g} \sigma c\left(R+\frac{1}{16} \alpha R^{2} \rho^{2}+\frac{1}{4} \alpha R \rho \square \rho-\frac{1}{2} \alpha R(\nabla \rho)^{2}+\frac{1}{4} \alpha \square \rho \square \rho\right)
$$

It does not contain $\tau$ at all. Nevertheless, the presence of $\tau$ was essential to have a solution to the consistency condition for the Weyl-variation of the metric, which did contain $\tau$. If, on the other hand, we had started with only $\tilde{O}$, which corresponds to setting $\alpha=0$, we would not have have found any non-trivial consistent anomaly to $\mathcal{O}(\tau)$.

We now discuss the special role of $\tau$. To begin with, a dimension four operator $\tilde{O}$ in $d=2$ with a linear coupling to energy-momentum tensors is very special: obviously the coupling should start with two energy-momentum tensors. Writing $\tilde{O}$ as the product of its left $\tilde{O}_{L}$ and right $\tilde{O}_{R}$ components, we need a non-zero correlator of $\tilde{O}_{L}$ with two left components of the energy-momentum tensor $T_{L}$. In $d=2$, due to the infinite dimensional Virasoro symmetry, the correlator is nonzero only if $\tilde{O}_{L}$ itself is proportional to $T_{L}$. A similar argument holds for the right component and we conclude that $\tilde{O}$ has to be identified with the famous $T_{L} T_{R}[14]$.

We can see this happen quite generally. In $d=2$ the contribution to the $\mathcal{O O}$ OPE from the vacuum representation, to which the energy-momentum tensor belongs, is fixed by the Virasoro symmetry to

$$
\begin{aligned}
\mathcal{O}(z, \bar{z}) \mathcal{O}(w, \bar{w}) & =\left(\frac{1}{(z-w)^{3}}+\frac{3}{c} \frac{1}{z-w} T_{L}(w)+\ldots\right)\left(\frac{1}{(\bar{z}-\bar{w})^{3}}+\frac{3}{c} \frac{1}{\bar{z}-\bar{w}} T_{R}(\bar{w})+\ldots\right) \\
& =\cdots+\frac{9}{c^{2}} \frac{1}{|z-w|^{2}} T_{L}(z) \bar{T}_{R}(\bar{z})+\ldots
\end{aligned}
$$

as one can explicitly verify for the free fermion model with $c=\frac{3}{2}$. The operator $\tilde{\mathcal{O}}=T_{L} T_{R}$ indeed appears in the OPE of two $\mathcal{O}$ operators with a logarithmic coefficient. The operator $\mathcal{O}$ has all its couplings determined by being a nonsingular product of energy-momentum tensors. In our scheme, which preserves diffeoinvariance, it can be given an explicit diffeoinvariant definition as

$$
\tilde{\mathcal{O}}=T_{\mu \nu} T^{\mu \nu}
$$


which differs from the original expressions by terms involving the trace $T_{\mu}^{\mu}$, which vanishes as an operator. In correlators the two definitions differ therefore by local terms. It is obvious that all the couplings of $\tilde{\mathcal{O}}$ are determined by the couplings of the energymomentum tensors and therefore it cannot generate anomalies with independent universal coefficients. We can analyze this further by studying the terms involving $\tilde{\mathcal{O}}$ to linear order in its coupling in the Osborn Equation. To order $\rho^{2}$ there is a term in the equation which in leading order has the form:

$$
\rho \log \frac{\square}{\mu^{2}} \rho\left\langle T_{\mu \nu}\right\rangle_{1}\left\langle T^{\mu \nu}\right\rangle_{1}
$$

originating in the product of the chirally factored correlators $\left\langle\mathcal{O}_{L} \mathcal{O}_{L} T_{L}\right\rangle\left\langle\mathcal{O}_{R} \mathcal{O}_{R} T_{R}\right\rangle$. Here $\left\langle T_{\mu \nu}\right\rangle_{1}$ is the expansion of the nonlocal energy-momentum tensor defined from the nonlocal Polyakov action, to first order around the flat metric, i.e.

$$
\left\langle T_{\mu \nu}\right\rangle_{1} \propto\left(\eta_{\mu \nu}-\frac{\partial_{\mu} \partial_{\nu}}{\square}\right)\left(\partial^{\alpha} \partial^{\beta}-\eta^{\alpha \beta} \square\right) h_{\alpha \beta}
$$

The term in (4.8) has an explicit logarithmic singularity and when in higher order more energy-momentum tensors are correlated, i.e. (4.9) is evaluated in the background of a generic metric $g_{\mu \nu}$. When acted upon by the standard metric variation the logarithmic singularity is eliminated but the powerlike singularities remain. In order to cancel them an explicit (non-local) term linear in $\tau$ should be introduced

$$
\int d^{2} x \sqrt{g} \tau(x)\left\langle T_{\mu \nu} T^{\mu \nu}\right\rangle_{g}
$$

where the matrix element of the energy-momentum tensors has to be evaluated in a generic background $g_{\mu \nu}$. This shows that without the explicit coupling linear in $\tau$, the Osborn Equation is not consistent to order $\rho^{2}$ and therefore we should expect that this is reflected on the metric beta-function. Indeed we found that consistency requires that it contains both $\rho$ and $\tau$. Now we discuss the second puzzle raised before, i.e. why the Weyl anomaly does not contain $\tau$-dependent terms. After the addition of (4.10) we should consider it together with the pure $g_{\mu \nu}$ dependent (Polyakov) term, i.e.

$$
\tilde{W}=-\frac{c}{4} \int d^{2} x \sqrt{g} R \frac{1}{\square} R+\int d^{2} x \sqrt{g} \tau(x)\left\langle T_{\mu \nu}\right\rangle_{g}\left\langle T^{\mu \nu}\right\rangle_{g}
$$

For the evaluation of the energy-momentum matrix element we used the fact that in a chiral scheme the correlator is factored into a "left" and "right" part, and therefore in a general background in the diff-invariant scheme it can be also evaluated in a factorized form. The result is valid modulo local terms. It is clear that the second term does not contain new universal information. More explicitly we can show to order $\tau$ that (4.11) obeys a homogenous Osborn Equation. The Weyl variation of $\tilde{W}$ at order $\tau$ is

$$
\delta_{\sigma} \tilde{W}=\int d^{2} x \sqrt{g} T^{\mu \nu}\left(\beta_{\mu \nu}^{g}+2 \tau \delta_{\sigma} T_{\mu \nu}\right)
$$


where $\beta^{g}$ is to be determined and the energy-momentum tensor are determined from the Polyakov action. Using

$$
\delta_{\sigma} T_{\mu \nu}=\frac{c}{4}\left(\nabla_{\mu} \nabla_{\nu} \sigma-g_{\mu \nu} \square \sigma\right)
$$

the second term leads in (4.12) to the term $\int \sqrt{g} \tau R \square \sigma$, which is cohomologically trivial, being the Weyl variation of $\int \sqrt{g} \tau R^{2}$. The first term is cohomologically non-trivial and would lead to a non-local expression for $\delta_{\sigma} W$. Requiring this to vanish determines $\beta^{g}$

$$
\beta_{\mu \nu}^{g}=-\frac{c}{2} \tau \nabla_{\mu} \nabla_{\nu} \sigma
$$

in agreement with (4.2), after rescaling $\tau$ appropriately.

We see that the $\tau$-dependent part of the metric beta-function is entirely determined by the Polyakov action and does not reflect any new universal coefficient of a logarithm. As a consequence there is no contribution to the anomaly. This is a very special feature of $d=2$ related to the fact that the $\Delta=4$ operator is the ubiquitous $T \bar{T}$ [14]. There is of course an ambiguity of local terms which could be added to the generating functional.

\section{Conclusions}

The main result of this paper is that integer dimension irrelevant operators change in a significant fashion the structure of the Osborn Equation. The presence of their sources in the generating functional requires a non-trivial beta-function for the metric, i.e. an addition to its classical transformation rule under Weyl rescaling which depends on the irrelevant sources. To any given finite order in the number of irrelevant sources the equation stays consistent and a local Weyl anomaly can be defined. It contains the information about the normalization of universal single logarithms in various correlators.

We now summarize the pattern for beta-functions and Weyl anomalies which we find in any even dimension $d(d=2$ having the special features discussed in Section 4$)$ :

a. Starting with an integer dimension $\Delta>d$ operator $\mathcal{O}$ with source $\rho$, there is a logarithmic singularity in the two-point funtion $\langle\mathcal{O} \mathcal{O}\rangle$ and a double logarithm in the correlator $\langle T \mathcal{O O}\rangle$.

b. As a consequence there is a metric beta function to order $\rho^{2}$.

c. The metric beta-function implies a deformation of the Weyl anomaly generated by the correlator of two energy-momentum tensors. The same anomaly contains the information about the two point function of $\mathcal{O}$, thus generalizing the type $\mathrm{B}$ anomalies of marginal and relevant operators.

d. The other Weyl anomalies involving energy-momentum tensors are deformed to order $\rho^{2}$ by the presence of the metric beta-function. They are related to decompositions of the two $\langle T \mathcal{O O}\rangle$ energy-momentum correlators, where the two $\mathcal{O}$ 's couple through the energy-momentum tensor conformal block. 
e. To order $\rho^{2}$ there are beta-functions generated for any integer dimensional $\tilde{\mathcal{O}}$ operator with dimension $\tilde{\Delta} \leq(2 \Delta-d)$ and coupling $\tau$. These beta-functions deform the type B anomalies which are linear in $\tau$. To $\mathcal{O}\left(\rho^{2}\right)$ the deformed Weyl anomalies are related to correlators of two $\mathcal{O}$ 's and energy-momentum tensors, where the two $\mathcal{O}$ 's couple to the energy-momentum tensors through the $\tilde{\mathcal{O}}$ conformal block.

An application of the formalism discussed in this paper is the study of correlators of elements of the chiral ring in $\mathcal{N}=2$ superconformal theories in four dimensions $[17,18]$. The chiral ring contains irrelevant operators and their correlators can be studied using the methods of this paper and the anomaly equations generalized to a supersymmetric set up. The powers of curvatures present in the anomaly formulae define the operator mixings involved. Moreover the anomaly coefficients can depend on constant moduli. We are currently studying this generalization in more detail.

A natural question is the physical meaning of the metric beta-function. In a CFT the metric acts as a source for the energy-momentum tensor and the beta-function is just a convenient way to organize the recursion relations connecting the various powers of the logarithms. When however the irrelevant couplings are turned on as true massive deformations of the CFT, the metric beta-function indicates that the metric considered as a coupling also starts running. A natural interpretation of the metric as a coupling is when the theory is formulated on a curved manifold: the parameters characterizing the geometry of the manifold can be considered to be coupling constants which multiply the terms in the Lagrangian involving energy-momentum tensors. In particular when the theory is formulated on $S_{d}$ the radius becomes a coupling constant in the above sense. We reach the strange conclusion that the radius of the sphere on which the theory is formulated has a nontrivial renormalization group transformation. The scale dependent contribution to the partition function following from the transformation of the radius enters through the anomaly: for the $\Delta=5$ theory in $d=4$ the "c" anomaly for constant $\rho$ vanishes on the sphere but not the " $a$ " anomaly, such that such a new type of dependence is possible in principle. The strange feature mentioned above depends of course on turning on the irrelevant perturbation which is not consistent outside a finite order of perturbation theory.

The structure of the anomalies corresponding to terms with multiple logarithms in the generating functional can be understood by choosing a decomposition of the correlators in conformal blocks where the two irrelevant operators couple first to an integer dimensional block. It is an interesting question how the logarithmic structures are reproduced by other decompositions related by crossing.

Acknowledgements: We thank E. Brehm, H. Godazgar, R. Manvelyan and E. Skvortsov for discussions. Some of the calculations were performed with the help of the xAct collection of Mathematica packages. This work was supported in part by I-CORE program of the Planning and Budgeting Committee and the Israel Science Foundation (grant number 1937/12) and by GIF - the German-Israeli Foundation for Scientific Research and Development (grant number 1265). 


\section{References}

[1] M. J. Duff, "Twenty years of the Weyl anomaly," Class. Quant. Grav. 11, 1387 (1994) [hep-th/9308075].

[2] S. Deser, M. J. Duff and C. J. Isham, "Nonlocal Conformal Anomalies," Nucl. Phys. B 111 (1976) 45.

[3] S. Deser and A. Schwimmer, "Geometric classification of conformal anomalies in arbitrary dimensions," Phys. Lett. B 309 (1993) 279 [hep-th/9302047].

[4] H. Osborn, "Weyl consistency conditions and a local renormalization group equation for general renormalizable field theories," Nucl. Phys. B 363 (1991) 486.

[5] I. Jack and H. Osborn, "Analogs for the $c$ Theorem for Four-dimensional Renormalizable Field Theories," Nucl. Phys. B 343 (1990) 647.

[6] I. Jack and H. Osborn, "Constraints on RG Flow for Four Dimensional Quantum Field Theories," Nucl. Phys. B 883 (2014) 425 [arXiv:1312.0428 [hep-th]].

[7] F. Baume, B. Keren-Zur, R. Rattazzi and L. Vitale, "The local Callan-Symanzik equation: structure and applications," JHEP 1408 (2014) 152 [arXiv:1401.5983 [hepth]].

[8] H. Baum and A. Juhl, "Conformal Differential Geometry", Birkhäuser 2010.

[9] J. Gomis, P. S. Hsin, Z. Komargodski, A. Schwimmer, N. Seiberg and S. Theisen, "Anomalies, Conformal Manifolds, and Spheres," JHEP 1603 (2016) 022 [arXiv:1509.08511 [hep-th]].

[10] D. R. Karakhanian, R. P. Manvelyan and R. L. Mkrtchian, "Trace anomalies and cocycles of Weyl and diffeomorphism groups," Mod. Phys. Lett. A 11 (1996) 409 [hep-th/9411068].

[11] A. Bzowski, P. McFadden and K. Skenderis, "Renormalised CFT 3-point functions of scalars, currents and stress tensors," JHEP 1811 (2018) 159 [arXiv:1805.12100 [hep-th]].

[12] H. Osborn and A. C. Petkou, "Implications of conformal invariance in field theories for general dimensions," Annals Phys. 231 (1994) 311 [hep-th/9307010].

[13] J. Erdmenger and H. Osborn, "Conserved currents and the energy momentum tensor in conformally invariant theories for general dimensions," Nucl. Phys. B 483 (1997) 431 [hep-th/9605009].

[14] A. B. Zamolodchikov, "Expectation value of composite field $T \bar{T}$ in two-dimensional quantum field theory," hep-th/0401146. 
[15] B. C. van Rees, "Irrelevant deformations and the holographic Callan-Symanzik equation," JHEP 1110 (2011) 067 [arXiv:1105.5396 [hep-th]].

[16] D. Z. Freedman, K. Johnson and J. I. Latorre, "Differential regularization and renormalization: A New method of calculation in quantum field theory," Nucl. Phys. B 371 (1992) 353.

[17] M. Baggio, V. Niarchos and K. Papadodimas, "On exact correlation functions in $\mathrm{SU}(\mathrm{N}) \mathcal{N}=2$ superconformal QCD," JHEP 1511 (2015) 198 [arXiv:1508.03077 [hep-th]].

[18] E. Gerchkovitz, J. Gomis, N. Ishtiaque, A. Karasik, Z. Komargodski and S. S. Pufu, "Correlation Functions of Coulomb Branch Operators," JHEP 1701 (2017) 103 [arXiv:1602.05971 [hep-th]]. 